\title{
The Self-organizing Seismic Early Warning Information Network (SOSEWIN)
}

\section{K. Fleming, ${ }^{1,4}$ M. Picozzi, ${ }^{1} C_{.}$Milkereit, ${ }^{1}$ F. Kühnlenz, ${ }^{2}$ B. Lichtblau, ${ }^{2}$ J. Fischer, ${ }^{2}$ C. Zulfikar, ${ }^{3}$ O. Özel, ${ }^{5}$ and the SAFER and EDIM working groups $^{1,2,3}$}

\section{INTRODUCTION}

Rapid urbanization, the interconnection of economies and increasing dependence on technology makes modern society ever more vulnerable to natural disasters. This is particularly true when considering the growth of so-called mega cities (defined by the United Nations as metropolitan areas with populations exceeding 10 million), the majority of which are in the developing world. This has led to the recognition of the importance of early warning systems as one means of mitigating the potential human and economic losses resulting from natural disasters (e.g., International Strategy for Disaster Reduction 2005; United Nations 2006).

Considering earthquakes, many areas of major urbanization (e.g., Tokyo, Istanbul, Naples, Mexico City) are exposed to significant seismic hazard. Currently, several parts of the world have some form of earthquake early warning system (EEWS) either in operation or under development (e.g., Taiwan: Wu and Teng 2002; Japan: Horiuchi et al. 2005; California:

1. Helmholtz-Zentrum Potsdam Deutsches GeoForschungsZentrum, Potsdam, Germany

2. Department of Computer Science, Humboldt-Universität zu Berlin, Berlin, Germany

3. Earthquake Engineering Department, Kandilli Observatory and Earthquake Research Institute, Bogaziçi University, Istanbul, Turkey

4. Department of Spatial Sciences, Curtin University of Technology, Perth, Western Australia, Australia

5. Faculty of Engineering, Istanbul University, Istanbul, Turkey

The SAFER and EDIM working groups developing the sensing node and operating the SOSEWIN test-bed network are J. Zschau, C. Milkereit, M. Picozzi, K. Fleming, I. Veit, K.-H. Jäckel, M. Hönig, J. Nachtigall, and H. Woith (Helmholtz-Zentrum Potsdam Deutsches GeoForschungsZentrum, Germany);J. Fischer,J.P. Redlich, B. Lichtblau, F. Kühnlenz, K. Ahrens, I. Eveslage, and S. Heglmeier (Department of Informatics, Humboldt University Berlin, Germany); and M. Erdik, C. Zulfikar, O. Özel, N. Kafadar (Earthquake Engineering Department, Kandilli Observatory and Earthquake Research Institute, Bogaziçi University, Istanbul, Turkey).
Wurman et al. 2007; Istanbul: Erdik et al. 2003; Bucharest: Ionescu et al. 2007; Mexico City: Espinosa-Aranda et al. 1995). However, these systems usually involve the use of a relatively low number of sensors (from several to tens of units), a fact largely dictated by the high cost of such instrumentation. In addition, these systems must usually communicate their data to centralized processing and archiving facilities. An example of a standard centralized EEWS is the Istanbul Earthquake Rapid Response and Early Warning System (IERREWS; Erdik et al. 2003) operated by Kandilli Observatory and Earthquake Research Institute (KOERI) of Bogaziçi University (Figure 1A). This system is made up of 10 strong-motion stations that are installed as close as possible to the fault zone of the north Anatolian fault, which runs through the Marmara Sea to the south of Istanbul. In the IERREWS, a centralized philosophy of early warning is adopted, meaning that there is a continuous telemetry of data between IERREWS stations and the main data center, where the alarm is decided. Transmission is realized through the use of a digital spread spectrum radio modem system involving repeater stations.

In contrast to such systems, the network envisaged in this paper is a new approach for early warning that has its origin in the vision of providing to the wider community a low-cost system relying on modern wireless technology (Figure 1B). This system, termed the Self-organizing Seismic Early Warning Information Network (SOSEWIN), will be characterized by the following features:

- Each seismological sensing unit or sensing node $(\mathrm{SN})$ is made of low-cost "off-the-shelf" components, with each unit initially costing several hundred euros, in contrast to thousands to tens of thousands for standard seismological stations.

- Each SN undertakes its own, onsite seismological data processing, preliminary analysis, archiving, and communication of data as well as early warning messages. Moreover, each $\mathrm{SN}$ will also have the capacity to measure other environmental parameters (e.g., noise, temperature, etc.).

- The reduced sensitivity of the SNs compared to standard instruments (due to the use of lower-cost components) will be compensated for by the network's density, which in 

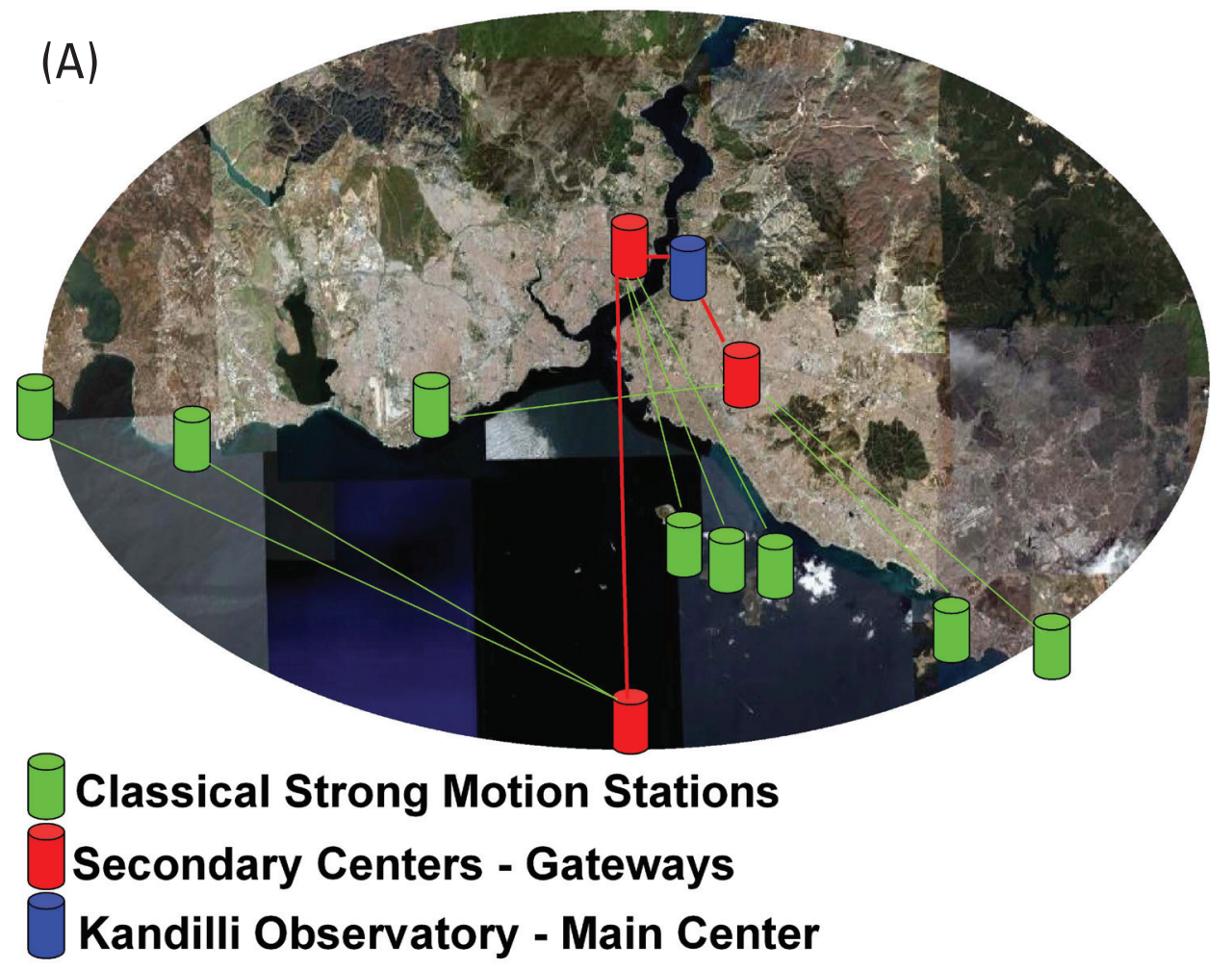

(B)

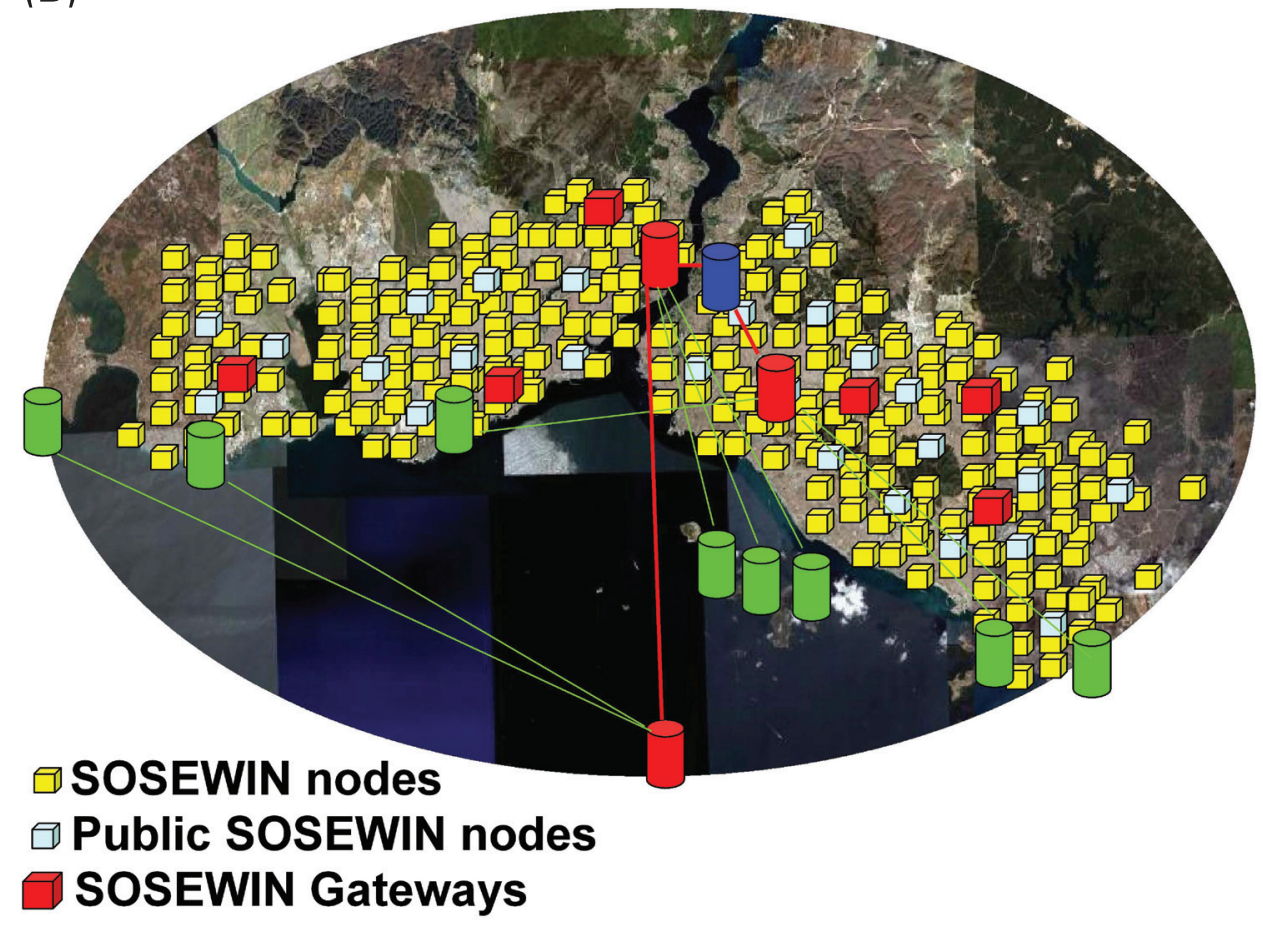

A Figure 1. How the SOSEWIN system compares with a standard network (in this case, the IERREWS, Istanbul). (A) A standard network consists of a relatively low number of stations that are linked to a central processing center, either directly or via another "gateway" station. (B) SOSEWIN, by contrast, will not require a centralized center, with the alerts being propagated throughout the system following an event's verification. The SOSEWIN nodes may also be part of a public warning system (blue boxes) or owned by members of the general public (yellow boxes). 
the future is expected to number hundreds to thousands of units over areas served currently by the order of tens of standard stations.

- SOSEWIN will be a decentralized, self-organizing ad-hoc wireless mesh network (WMN).

- The early-warning decision-making is carried out within the WMN of sensing units, taking advantage of their communication capability and the design of suitable alarming processes. Thus, the alarming itself can be done both inside the network (i.e., flooding the alarm to every node) and outside it (i.e., routing the alarm to the nearest gateway node, and then toward some external administrative center).

- Its self-organizing capability will allow it to adapt continuously to changing circumstances, e.g., the addition/ removal/malfunctioning of nodes, interference in communications due to local (and possibly time-varying) phenomena, loss of sections of the network following an earthquake, etc.

- Instruments will also be purchasable by the public. Thus, SOSEWIN also be able to integrate additional data from private persons.

- For rapid response purposes during the post-event period, the much higher instrumental density of SOSEWIN means tools such as ShakeMap (Wald et al. 2006) can rely more on real data and less on interpolation schemes.

Although wireless seismic networks have been proposed for high-density data acquisition (e.g., Savazzi and Spagnolini 2008), with lower-cost wireless-linked methodologies being deployed with some degree of self organization (e.g., WiLSoN, the "wirelessly linked seismological network"; Husker et al. 2008), SOSEWIN is believed to be the first such network employed for early warning, although wireless networks have been incorporated into other strong-motion networks for earthquake mitigation purposes (e.g., Evans et al. 2005). In addition, other low-cost seismometers, e.g., the "home seismometer" of Horiuchi et al. (2009), are being considered to complement existing early warning networks.

The development of SOSEWIN focuses on two points. The first is the design of the low-cost SNs themselves, while the second is its self-organizing, decentralized character. The originality of both these aspects of SOSEWIN will distinguish this network from more traditional types. Since SOSEWIN is concerned only with larger, potentially damaging events, the lower sensitivity expected from the SNs compared to standard commercial instruments is not a significant concern. Moreover, we wish to state immediately that SOSEWIN is not meant to replace existing networks, but to complement them.

This work describes the current state of the development of SOSEWIN and what we can expect its contribution to earthquake early warning (EEW) to be. The next section describes the general organization of SOSEWIN, including a description of the different types of units that are being developed to form a future operational system. This is followed by sections outlining the hardware that makes up the individual seismological sensing units, the network's seismological processing, the alarm/alert chain, the planned organizational and routing protocols, and the data archiving. We then outline the initial test deployment of SOSEWIN, which consists of a network of 20 SNs in the Ataköy district of Istanbul, Turkey. We conclude with a discussion of this network's wireless communication performance and the proposed next steps.

\section{GENERAL ORGANIZATION}

The high instrumental density of a network such as SOSEWIN has the advantage of an element of redundancy. However, this raises a number of challenges in how to ensure the most efficient management of the network, keeping in mind that it is a decentralized system. One issue is that by the very nature of an earthquake early warning system (EEWS), communications traffic would be, most of the time, relatively low (i.e., "housekeeping," ensuring that all elements of the network can be reached and are in contact with those entities that would require the warning). However, if an event occurs, then there will be a near instantaneous rise in communications, potentially requiring alerts to be sent to all sensors. Therefore, the most efficient means of communicating the warning, while at the same time verifying the event, must be employed. For these reasons, the general arrangement of SOSEWIN is characterized by a two-level hierarchical architecture consisting of an upper application layer and a lower communication layer (Figure 2).

The lowest level of the application layer is made up of SNs that are divided into groups termed clusters. Clusters are the fundamental organizational unit of SOSEWIN, and each of them is headed by a so-called leading node (LN). The LNs are effectively the same unit as the SNs, but while they have the same hardware, they temporarily carry out the roles of coordination and decision. The aim of this sublayer is to regulate the activities of the SNs within their clusters, based on criteria specified for the cluster as a whole (e.g., the number of $\mathrm{SNs}$ that lead to a group alarm) and the individual SNs (e.g., event trigger thresholds). Each $\mathrm{SN}$ within a cluster communicates general "housekeeping/status" information, ground motion parameters, and alarms to its LN. Which SN belongs to which cluster, and which node serves as the LN, will be defined by a clustering algorithm where the main constraint is to maintain the optimal communications capability, as defined by the wireless metric parameter (WMP, see below). By this, we mean that each $\mathrm{SN}$ is able to communicate in the fastest way (e.g., minimizing the number of steps a given message must make from node to node, termed K-hops) with the least interference and data loss with its LN, and likewise for the LN to communicate with its neighboring LNs. One constraint that has been decided upon is that an $\mathrm{SN}$ within a cluster is at the most two $\mathrm{K}$-hops from its LN. The higher sublayer of the application layer is the critical one in terms of distributing alarms among LNs and issuing them to end users, which also, naturally, requires the use of highly efficient communications protocols. This layer in turn may, as the number of nodes increases, be structured into additional sublayers; for example, it might be necessary 


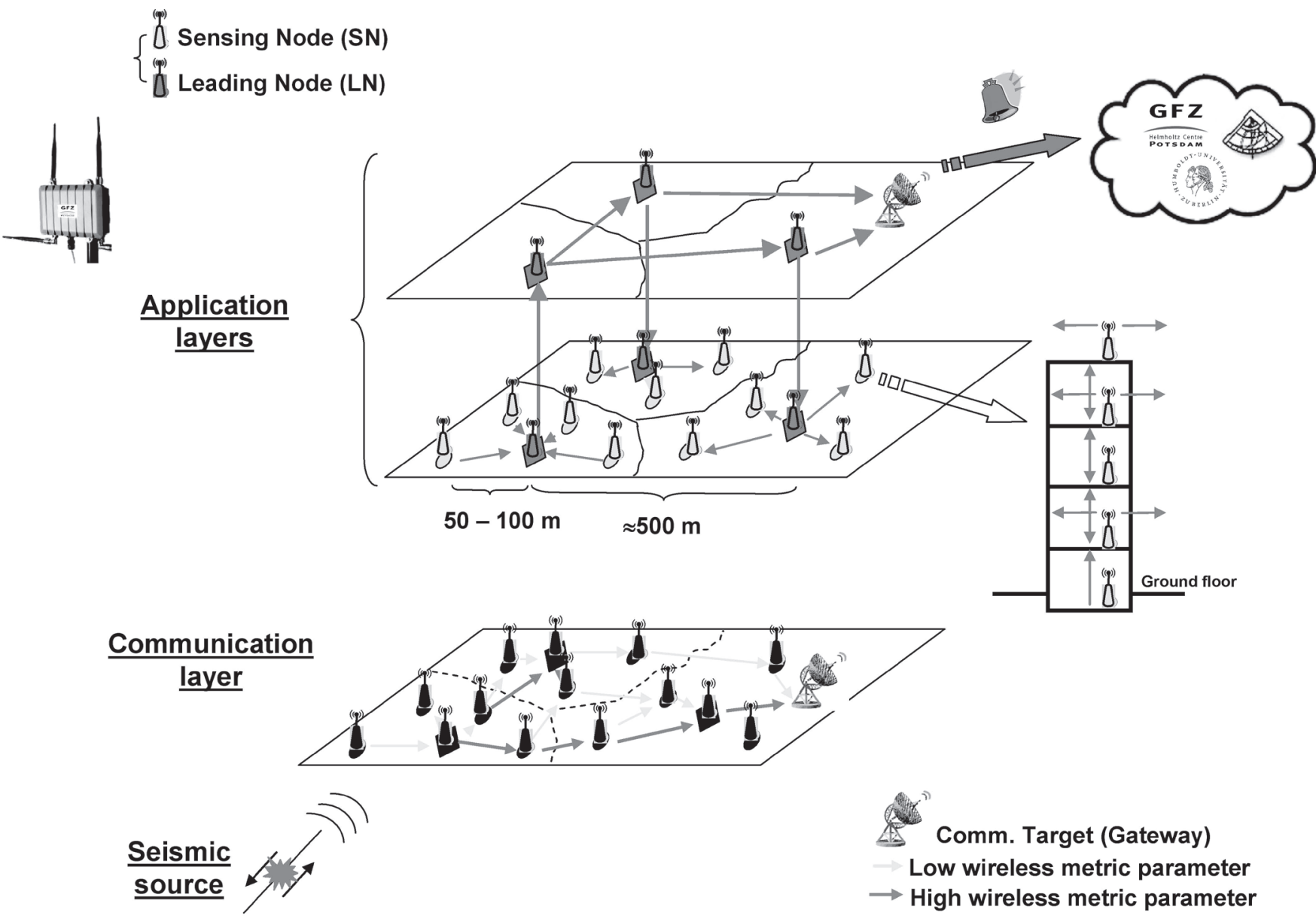

A Figure 2. The general organization of SOSEWIN. The wireless metric parameter is a measure of the communications efficiency of the network. Note the subdivision of the network into clusters (solid boundary in the application layers, dashed boundary in the communication layer). The applications layer may also be divided into sublayers as the number of nodes increases. The sensors may be distributed individually or as a series within a building (right-hand cartoon from the lower application layer). While the system is decentralized, gateways will still be required to communicate alerts and other information to outside the network.

to define a "leader of leading nodes" layer. Finally, there is a communication layer, where the clustering organization will operate and there is peer-to-peer communications between the nodes. In other words, in this layer, there is not a role distinction among the nodes, and the routing of data and warning messages toward a target are regulated by a dedicated protocol.

As currently planned, the distances involved are of the order of 50 to $200 \mathrm{~m}$ between SNs, leading to separations of 200 to $1,000 \mathrm{~m}$ between neighboring LNs (given the two K-hop constraint). While geometrical/seismological constraints (shape and areal extension of each cluster, number of $\mathrm{SNs}$ within a cluster, etc.) are also used as organizational criteria, they are secondary to optimizing the communications.

When necessary, the clustering algorithm will reorganize the network to ensure the optimal communications capacity. This can happen when, for example, there is a malfunction in an LN or when the WMP drives the network to obtain an improved cluster configuration if, for example, additional nodes are added. However, it is essential (and an imposed condition) that the cluster configuration does not change when an event has been detected, or rather, those clusters that are aware of an earthquake having occurred cannot alter their communications arrangement.

It is planned that apart from the $\mathrm{SNs}$ and $\mathrm{LNs}$ already discussed, several other types of seismological sensing units (Table 1) will be added to SOSEWIN to broaden its operational capacity. In particular, the main nodes (MN) and gateways (GN) are SNs with additional capability. In the case of the $\mathrm{MN}$, this will involve more computing power and data storage capacity, while for the GN, this involves additional communications hardware, e.g., Internet connections, satellite phones, etc. However, if circumstances require it, MNs and GNs may serve as LNs. Another type is the external node (EN), which, while possibly a seismic station of some sort, is not actually part of SOSEW IN but is in communication with SOSEWIN. One may therefore think of the GN as being an SN that communicates with an entity outside the network, such as a processing center, on the terms of that entity (e.g., via the Internet or another wireless system), while the EN is an entity that communicates with SOSEWIN from outside the system on SOSEWIN's terms, or it may be the entity communicated to by the GN. Finally, there are temporary nodes (TN), which 


\begin{tabular}{|c|c|}
\hline \multicolumn{2}{|r|}{$\begin{array}{c}\text { TABLE } 1 \\
\text { The Different Seismological Sensing Unit Types That Will Make up a Future Operational SOSEWIN }\end{array}$} \\
\hline Sensing Node (SN) & $\begin{array}{l}\text { The primary unit of the network. Monitors ground motion, undertakes data analysis and event detec- } \\
\text { tion, and communicates with its leading node (LN). }\end{array}$ \\
\hline Leading Node (LN) & $\begin{array}{l}\text { s. An SN is nominated as an LN based first on communications } \\
\text { ological and geometrical considerations. }\end{array}$ \\
\hline Main & 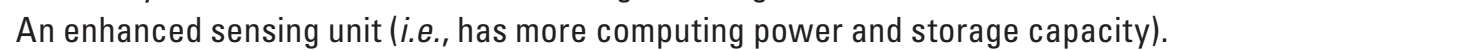 \\
\hline Routing Node (RN) & e of the network and is able to choose a route for the most \\
\hline Temporary Node (TN) & Is mainly for access to the network (e.g., a field laptop) to obtain additional seismological data. \\
\hline Node (EN) & Is only in communication with our \\
\hline Gateway (GN) & $\begin{array}{l}\text { Is an SN with extra components for communicating outside the network, for example with the Internet } \\
\text { or other networks or processing centers, e.g., via satellite. }\end{array}$ \\
\hline
\end{tabular}

would usually take the form of a laptop connected to any node that belongs to the network (and is in wireless communications range). For a certain time period, the TN therefore becomes part of SOSEWIN and is able to query and manage all nodes. Such a situation would arise, for example, during general network maintenance or after an event when waveform data are being transferred from the nodes to a laptop for future, more detailed analysis. It should also be noted that, assuming that node's wireless communications are still operational, the TN will be able to download the required waveform data remotely without visiting the actual site, thus allowing the safe retrieval of data from damaged buildings.

\section{SN'S HARDWARE AND SOFTWARE}

Figure 3 outlines the main components of an SN, with some technical details listed in Table 2. The SN is divided into three parts, namely:

- The sensors;

- The digitizer board;

- The wireless router applications platform (WRAP) board. All components are bought off-the-shelf, with the exception of the digitizer board's analog-digital converters (ADC), which have been developed within the Deutsches GeoForschungsZentrum (German Research Center for Geosciences, GFZ), leading to the $\mathrm{SN}$ s being much less expensive (about 600 euros per unit) than standard seismometers.

The sensors include three accelerometers arranged to provide three-component $(\mathrm{X}, \mathrm{Y}$, and $\mathrm{Z})$ data and an additional sensor to measure an environmental parameter, such as noise, temperature, water pressure, etc. The accelerometers are based on MEMS (micro-electromechanical systems) sensors, which were originally designed to serve as controllers for automobile air bag safety units but have also been successfully incorporated into various seismic networks (Holland 2003; Evans et al. 2005; Wu and Kanamori 2008) and are proposed to be exploited for oil and gas exploration (e.g., Hons et al. 2008). The units used in the SNs have a measurement range of $\pm 1.7 \mathrm{~g}$, with a bandwidth of $25 \mathrm{~Hz}$ and a noise level of $0.5 \mathrm{mg}$.

The digitizer board for the $\mathrm{SN}$ prototype consists of four analog-digital converters (ADC) that sample the three-compo- nent accelerometers and the environmental sensor, a GPS unit that provides time and geographical coordinates, and a USB interface (Figure 3C). The ADC has a resolution of 24 bits, effectively providing a resolution of 19 bits. The sample rate is variable between 50 to 400 samples per second (sps), with 100 sps currently used. The USB chip combines the readings from the ADC units and the GPS device and sends them in two streams (one for the sensor data, one for the GPS) to the WRAP board.

The sensor data record consists of the four digitized sensor channel readings, a sample counter, a special start byte (which also identifies the first sample in every second, synchronized by the GPS device) and a CRC-8 checksum. The GPS device can output NMEA-0183 (standard of the National Marine Electronics Association, an ASCII format) or SiRF binary format (SiRF Technology Inc, http://www.sirf.com). While ideally all SNs should have GPS, some may not be able to use it, for example, an $\mathrm{SN}$ installed in a basement. This brings up one of the difficulties in designing SOSEWIN: the sometimes incompatible requirement for good coupling between the sensor and the ground for accurate ground motion measurements, and access to clear communications. Hence, there will be procedures whereby an SN without GPS timing will be able to synchronize itself with one that does, e.g., the basement $\mathrm{SN}$ can synchronize itself with a unit on the roof.

The WRAP board uses a 32-bit x 86 processor at $233 \mathrm{MHz}$, generally comparable with the performance of a classical 486er CPU. This embedded PC with a $266-\mathrm{MHz}$ CPU runs the Linux OpenWRT operating system. It has three roles: (1) analysis of the data, (2) storage of the data, and (3) communications of both raw data and computed parameters. There is one slot for a CompactFlash card which acts as the hard disk (currently 2 GB in size, but easily increased), and two positions for WLAN (wireless local area network, $2.4 \mathrm{GHz}$ or $5 \mathrm{GHz}$ ) Mini PCI cards. In addition, it has a power supply plug, two USB connectors, a serial port, and $100 \mathrm{MBit} / \mathrm{s}$ Ethernet. Supplying power to the WRAP board over the Ethernet interface using PoE (power over Ethernet) is also possible. All boards are installed in waterproof outdoor metal cases. Omni-directional dualband antennas with a gain of $5 \mathrm{~dB}$ are mounted with opposite vertical polarization. The amount of power required by an $\mathrm{SN}$ 
(A)

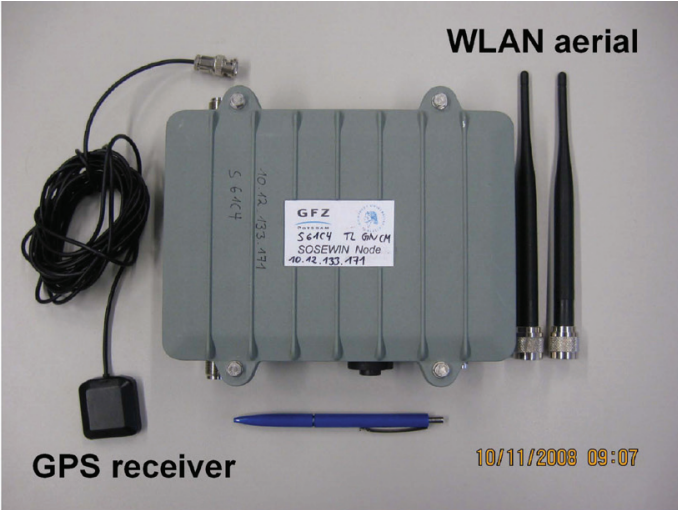

(B)

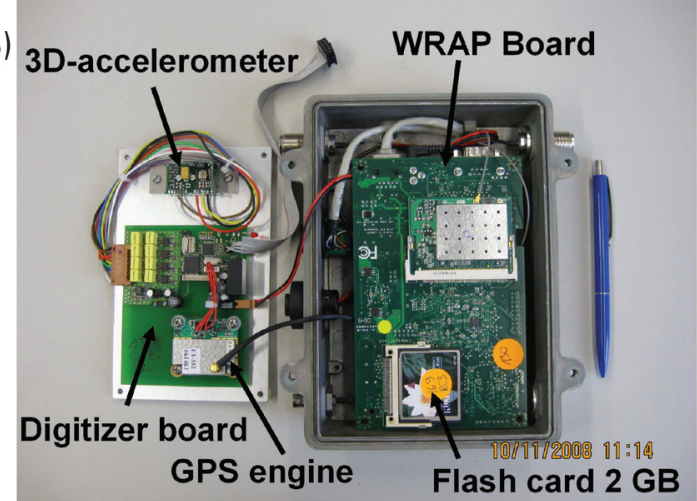

(C)

Digitizer board

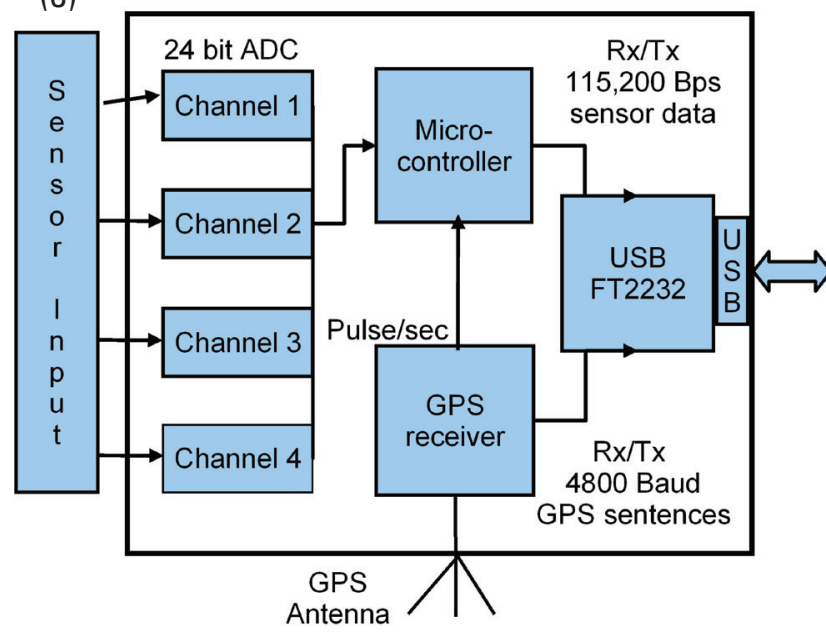

WRAP board

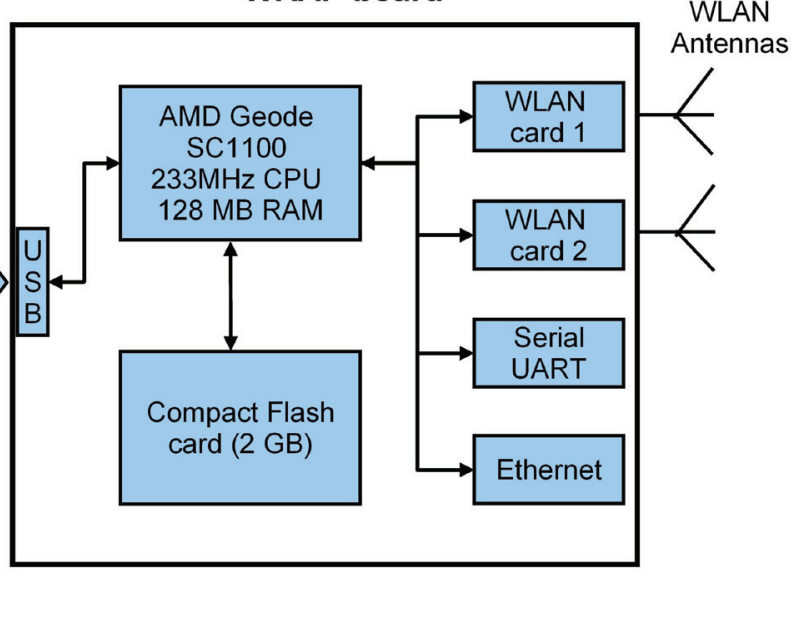

A Figure 3. The prototype sensing node (SN): (A) the complete unit and (B) an internal view. (C) A schematic overview of the architecture of the SN. Technical details of the various components are listed in Table 2.

when all operational activities are being undertaken (recording and real-time communication of data) has been experimentally measured to be about $4 \mathrm{~W}$.

The software operating on the SN currently consists of the following:

- OpenWRT: The operating system for the WRAP boards (http://www.openwrt.org) with Linux kernel 2.6.22 (Torvalds 2007). OpenWRT is an open-source, freely available, and highly configurable distribution. By default, it contains only the minimum that is required to run Linux, so it can also run on very size-limited systems. Moreover, it provides an environment for building your own Linux distribution for several platforms, including our x86er target platform for the WRAP boards.

- Data-provider: The program that handles the data streams from the digitizer board and then archives them via SeisComP/SeedLink.

- SeisComP/SeedLink: A software package and concept for near real-time seismic data distribution (http://geofon. gfz-potsdam.de/geofon//seiscomp/seedlink.html) developed by the GFZ. The SeedLink protocol is based on TCP (Heinloo 2000), and data are sent in the form of 512-byte
Mini-SEED packets with an 8-byte SeedLink header. The header contains the packet sequence number, which allows the unit to resume transmission where it left off (i.e., recovering the connection in the event of network errors and the support of non-permanent connections such as "dial-up mode"). It has a client-server architecture and is capable of tasks such as data acquisition, data recording, monitoring and controlling, real-time communications, user access, and near real-time data processing. In the $\mathrm{SN}$, the SeedLink server stores the data in a ring buffer of configurable size on the Compact Flash card. The data in the ring buffer will be kept for 20 days before it is overwritten. If more storage is needed, a larger Compact Flash card can be used.

- Optimized link state routing: OLSR is a table-driven, proactive routing protocol currently chosen for the WMN (http://www.olsr.org). As a proactive protocol, it periodically assesses and maintains the network topology by flooding information about its direct neighborhood throughout the whole network. OLSR has proven that it is capable of operating with hundreds of nodes and is widely accepted by several mesh networking communities, i.e., 
TABLE 2

Technical specifications of the various components that make up the sensing nodes as currently used in SOSEWIN

Accelerometers (MEMS ADXL203 chip)

Bandwidth

Sensitivity

Measurement range

Output noise

Digitizer board

Number of channels

$A D$ converter resolution/effective resolution

Input voltage range

Input impedance

Bit weight

Sample rate

Signal bandwidth $(-3 \mathrm{~dB})$

Stop bandwidth attenuation

Analogue anti-alias filter

Timing

Timing accuracy

Digital output

Temperature range

Power supply

Power consumption

WRAP board

CPU

DRAM (dynamic random access memory)

Operating system

Storage

Power consumption

Safety features

User interface

Possible expansions

Connectivity

BIOS
$25 \mathrm{~Hz}$ (up to $2.5 \mathrm{kHz}$ )

$1 \mathrm{~V} / \mathrm{g}$

$\pm 1.7 \mathrm{~g}$

$0.5 \mathrm{mg}$ (rms) at $20 \mathrm{~Hz}$

4

$24 / 19$ bits

$\pm 5 \mathrm{~V}$

$50 \mathrm{k} \Omega$

$0.6 \mu \mathrm{V}$

50 to 400 sps, standard is 100 sps

$20 \mathrm{~Hz}$

$>80 \mathrm{~dB}$ at $80 \mathrm{~Hz}$ or higher

$2^{\text {nd }}$-order $30-\mathrm{Hz}$ low-pass Butterworth

GPS NMEA messages and PPS

\pm 5 msec at 100 sps

USB ( $2 x$ virtual com-port, 115 kBaud data/4800 baud GPS)

$-20^{\circ}$ to $+70^{\circ} \mathrm{C}$

$+5 \mathrm{~V}$ (USB) or 9 to $18 \mathrm{~V}$ (on board DC/DC converter)

$50 \mathrm{~mA}$ at $5 \mathrm{~V}$ plus 70MA at $5 \mathrm{~V}$ for the GPS module

$233 \mathrm{MHz}$ AMD Geode SC1100 (486er core)

128 MB SDRAM ( synchronous dynamic random access memory)

CompactFlash card, currently $2 \mathrm{~GB}$

3 to $5 \mathrm{~W}$ at $12 \mathrm{VDC}$ (excluding miniPCI cards)

Watchdog timer built into the CPU, LM77 thermal monitors

3 front leds, console $1 / 0$ redirected to serial port

LPC bus for adding more serial ports, ISA style I/0, GPIO and I ${ }^{2} \mathrm{C}$ bus

1 Ethernet channel (National DP83816), 2 miniPCI slots, 1 serial port tinyBIOS version 1.11
Freifunk (http://www.freifunk.net) and the Funkfeuer (http://www.funkfeuer.at) projects

Additional secondary software includes MadWifi version 0.9.3 (http://madwifi.org) as the WiFi driver. MadWifi's regdomain setting was changed to the ETSI domain in order to obtain 13 channels for $802.11 \mathrm{~b} / \mathrm{g}$ (http://madwifi.org/wiki/UserDocs/ CountryCode).

\section{SEISMOLOGICAL PROCESSING}

SNs are developed with the primary goal of performing realtime seismological analysis for EEW. Considering the early warning requirement of issuing ground-motion estimates as quickly as possible, and the fact that $\mathrm{SNs}$ are composed of low-cost components, the general scheme designed for realtime processing involves the local, relatively simple, rapid, and robust analysis of data (Figure 4). However, before the seismological processing proceeds, the data is copied to a ring buffer contained on the flashcard (as mentioned before, about 20 days' worth on a 2-GB card). In addition, during the different stages of the seismological analysis (i.e., idle monitoring status, event detection, event characterization, and summary of the recorded ground motion during the event) the $\mathrm{SN}$ will issue within SOSEWIN a variety of short messages (i.e., a few hundred bytes) containing both seismological and engineering parameters (Table 3 ).

The sequence of the processing undertaken by the SN starts with the filtering of the accelerometer data using a 4thorder bandpass $(0.075$ to $25 \mathrm{~Hz}$ ) Butterworth filter (e.g., Wu and Kanamori 2005), which is followed by its integration using the recursive formulation of Kanamori et al. (1999) to provide real-time velocity and displacement. During the idle monitoring stages, messages will be regularly transmitted by the $\mathrm{SN}$ to its corresponding $\mathrm{LN}$ (minimizes traffic). These messages will consist 


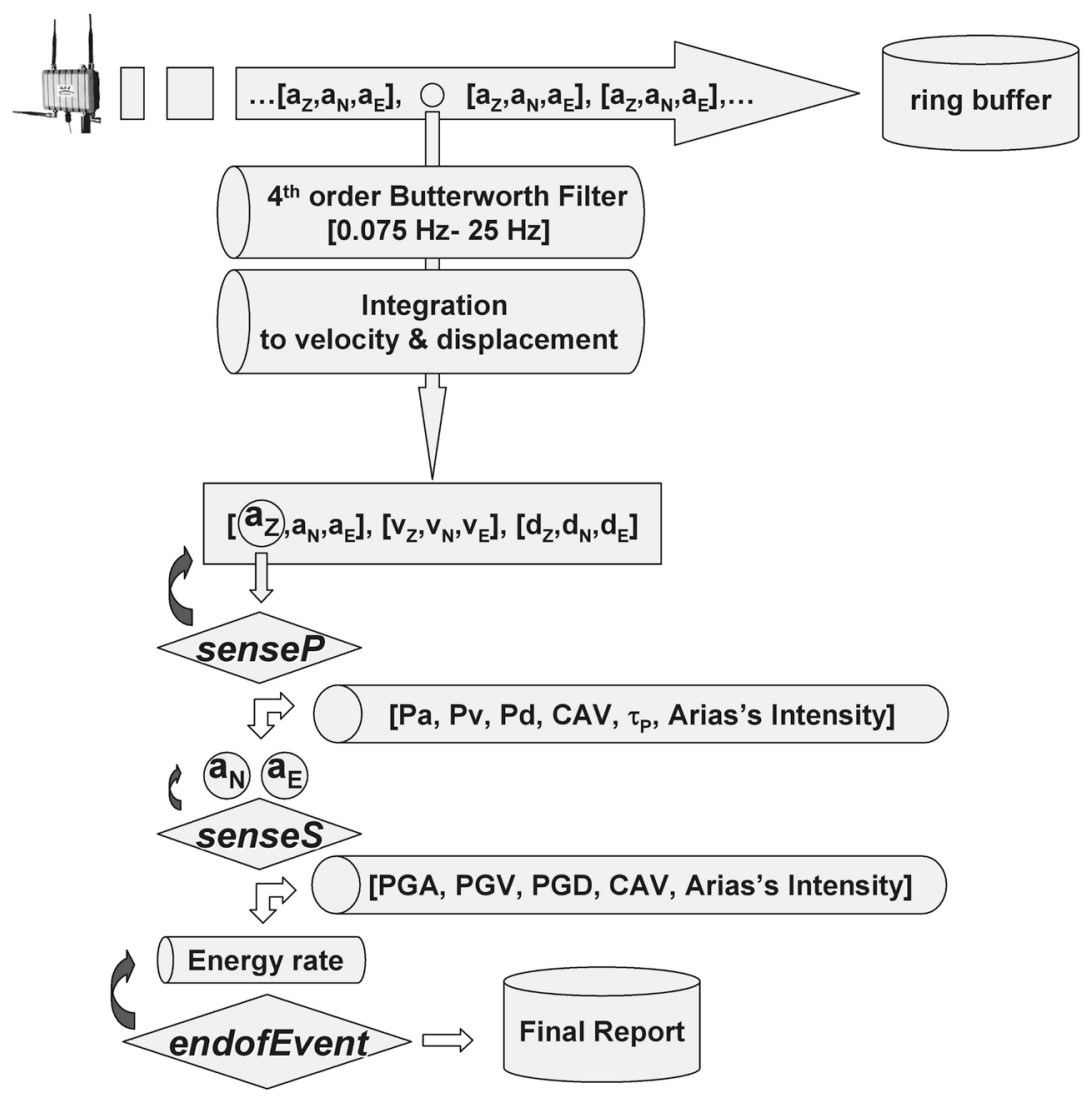

$\Delta$ Figure 4. The general scheme followed for the seismological processing and analysis that is being incorporated into the sensing nodes of SOSEWIN. Dark gray arrows indicate the continuous analysis of the data stream, while the white arrows show the analysis steps following the trigger of seismic phases or the end of the event.

of average values for the recorded acceleration, allowing onsite environmental seismic noise characterization, together with parameters indicating the health of the instrument (Table 3).

The most important seismological processing then undertaken is the detection of the earthquake's initial $P$ waves. As discussed by Wurman et al. (2007), in principle triggering can be performed using any real-time algorithm, under the constraint that it cannot be done with any method that requires data after the trigger itself, since by definition such data is unavailable at the time of the trigger. Therefore, methods such as autoregressive pickers (Sleeman and van Eck 1999) and pickers based on wavelet transforms (Zhang et al. 2003), are not practical for early warning applications even though they are more precise than a simple short-term/long-term average (STA/LTA) method.

For this reason, the procedure adopted within SOSEWIN is the STA/LTA method, which relies on the ratio between the average recorded absolute vertical ground motion component over a short time average window and that for a longer time average window, resulting in the STA/LTA or signal-to-noise ratio for $P$ waves (SNRP; Schweitzer et al. 2002; Trnkoczy 2002). An $S N$ is thus triggered when the SNRP exceeds a predefined value, which must be set, as well as the STA and LTA width, for each station to best suit the local environmental seismic noise conditions. We employ the recursive formulation developed by NORSAR (Schweitzer et al. 2002; Trnkoczy et al. 2002), where the SNRP is updated at every data point.

Immediately after triggering, the $\mathrm{SN}$ transmits the $P$-wave trigger time together with parameters that allow some indication of the severity of the ground motion, namely the peak ground motion parameters (peak ground acceleration $\mathrm{Pa}$, peak ground velocity $\mathrm{Pv}$, and peak ground displacement $\mathrm{Pd}$ ) for the vertical component of ground motion (Figure 4). In addition, the cumulative absolute velocity (CAV; Böse 2006), the Arias intensity (Arias 1970), and the predominant period $\left(\tau_{\mathrm{p}}\right.$; Olson and Allen 2005) will be calculated and included in these messages. From the moment of the trigger, all of these parameters will be continuously determined.

At the same time, the $S$-wave trigger will be activated. In practice, this involves the algorithm starting to check the ground motion on the horizontal components for the identification of the incoming $S$ waves. This operation is carried out using the LTA value frozen at the moment of the $P$-wave trigger and the STA computed from that moment using the vector-sum of the horizontal components. Therefore, $S$ waves are identified in a manner similar to the $P$ waves, when a predefined value of the signal-to-noise ratio for $S$ waves (SNRS) is exceeded. At this point, the event characterization stage is started with the computation and transmission of the $S$-wave trigger time, together with ground motion parameters (i.e., PGA, PGV, and PGV) continuously determined for the three components of 


\begin{tabular}{|c|c|}
\hline \multicolumn{2}{|r|}{$\begin{array}{c}\text { TABLE } 3 \\
\text { Messages Transmitted by an SN during the Different Stages of the Seismological Analysis }\end{array}$} \\
\hline SN status & Content of the message \\
\hline \multirow{6}{*}{ Idle } & SN MAC_-address \\
\hline & DateTime from GPS clock \\
\hline & Seismic noise for the three components of motion \\
\hline & Voltage, level of available energy in the battery \\
\hline & 4th channel (e.g., volume, temperature, etc.) \\
\hline & Geographical coordinates if there is a position change \\
\hline \multirow[t]{9}{*}{ Event Detection } & SN MAC—address \\
\hline & ID event \\
\hline & $P$-wave trigger time $\left(P t_{0}\right.$, in sec. $)$ \\
\hline & For the vertical component of ground motion: \\
\hline & $P$-wave peak ground acceleration $\left(P_{a}\right)$ and delay time from $P t_{0}$. \\
\hline & $P$-wave peak ground velocity $\left(P_{v}\right)$ and delay time from $P t_{0}$. \\
\hline & $P$-wave peak ground displacement $\left(P_{d}\right)$ and delay time from $P t_{0}$. \\
\hline & $\begin{array}{l}\text { Predominant period } \\
\text { CAV }\end{array}$ \\
\hline & Arias intensity \\
\hline \multirow[t]{10}{*}{ Event Characterization } & SN MAC—address \\
\hline & ID event \\
\hline & $P$-wave trigger time $\left(P t_{0}\right.$, in sec. $)$ \\
\hline & $S$-wave trigger time $\left(S t_{0}\right.$, in sec.) \\
\hline & For each component: \\
\hline & $S$-wave peak ground acceleration (PGA) \\
\hline & $S$-wave peak ground velocity (PGV) \\
\hline & $S$-wave peak ground displacement (PGD) \\
\hline & CAV \\
\hline & Arias intensity \\
\hline \multirow[t]{9}{*}{ Final Report } & SN MAC_-address \\
\hline & ID event \\
\hline & $P$-wave trigger time $\left(P t_{0}\right.$, in sec. $)$ \\
\hline & $S$-wave trigger time ( $S t_{0}$, in sec.) \\
\hline & Expiring time of the event (sec.) \\
\hline & For each component: \\
\hline & PGA, PGV, PGD \\
\hline & Arias intensity \\
\hline & Acceleration response spectra for the periods $0,1 \mathrm{~s}, 1 \mathrm{~s}$, and $3 \mathrm{~s}$ \\
\hline
\end{tabular}

ground motion (Figure 4). Naturally, the required parameters such as the $S$-wave trigger threshold will need to be defined for each site, while relations between magnitude and the results of the characterization calculations will be inferred over time as data is acquired by test SOSEWIN deployments.

To know when an earthquake has finished, or rather, when the most severe ground shaking has ended, we calculate the energy of the event, expressed as the integral of the squared velocity (Kanamori et al. 1999). Because energy is a monotonically increasing function, it is determined for a defined time interval (e.g., 1 second) and then reset to zero. This effectively gives the rate of the energy expended in the ground shaking; therefore, when the calculated value has decreased to $5 \%$ of the maximum that had been determined, the event is considered over.

The final actions involve the computation of the acceleration response spectra for some significant periods (i.e., $0.1 \mathrm{~s}, 1$ $s$, and $3 \mathrm{~s}$ ), and generating event files containing the summary of the peak ground motion values recorded during the event (PGA, PGV, and PVD). Finally, this information is incorporated into files (i.e., "Final Report" in Figure 4) that are produced in a format appropriate for the U.S. Geological Survey (USGS) tool ShakeMap (Wald et al. 2006).

Finally, while real-time event location is not included in the current version of the seismological analysis carried out by 
SOSEWIN, we intend to incorporate this in the near future. As more stations are triggered, these estimates will have to be continuously updated.

\section{ALARM AND ALERTING PROCESS}

As opposed to other standard early warning systems where the decision to send an alarm is centralized, that is, the decision is made at a main station outside the network, SOSEWIN employs a decentralized decision-making approach. This means that, thanks to its WMN nature, $P$-wave trigger information retrieved by SNs will be compared and analyzed by the SNs themselves, making it possible to rapidly and automatically make the decision cooperatively within the network about whether to issue an alarm. As stated before, because of the redundancy of information, it is necessary to have the system pass through several levels before the event is considered verified, i.e., before networkwide alerts are issued, since the issue of false and missed alarms is of serious concern to planners and developers of EEWS (e.g. Iglesias et al. 2007).

The general scheme of the alerting and alarm process planned for SOSEWIN will consist of four levels.

- Idle, where "all is well," i.e., sufficiently severe ground shaking has not been detected;

- $S N$ alert, where an individual sensor within a given cluster has detected strong enough ground shaking to inform its LN (while this may in fact be the LN itself, because of the geometry of the clusters, it would most likely be one of the other $\mathrm{SNs}$ );

- Group alert, where within a given cluster, $\geq 50 \%$ of the SNs have triggered;

- System alert, where $\geq$ three LNs have verified that an event has occurred, with a networkwide alert now issued.

This scheme may be graphically described by the so-called state machines of the SNs and LNs (Figure 5), which summarize the various stages each unit will pass through during an earthquake. The $\mathrm{SN}$ state machine is strongly related to the seismological activities of the SN, describing an SN's behavior resulting from variations in the level of ground motion. The state machine of the LN, on the other hand, outlines the decision-making/alarm-issuing actions that involve, at a higher level with regard to a single $\mathrm{SN}$, the whole network.

For the SN (Figure 5A), the various states are summarized as follows:

- State SN0 is the default or monitoring state, and involves the continuous processing of the accelerometric data, as outlined earlier.

- State SN1 is the event detection state (arrow A). The SN communicates this to its $\mathrm{LN}$ (arrow B), and from this point on, the event characterization (CAV, Arias, predominant period, energy rate, etc.) and $S$-wave detection algorithms start. However, if after a certain time (the expiring alert time, dependent upon the spacing of the $\mathrm{SNs}$ ) fewer than $50 \%$ of the $\mathrm{SNs}$ in the cluster trigger, the LN informs the $\mathrm{SN}$ to return to state $\mathrm{SNO}$ (arrows $\mathrm{C}$ and $\mathrm{D}$ ). This is then noted by the $\mathrm{SN}$ as a false alarm in an event file.

- State SN2 is the seismic event characterization state, which is reached either after SN1 (arrow E) or directly from SN0 if directed to do so by its LN (arrow F). Although event characterization has already started, this state is treated separately, since in $\mathrm{SN1}$, it is believed that "something may have happened," while here "something has happened."

- State SN3 is the disaster management state, and is reached after the event is considered over (arrow G). Activities here include creating the peak ground motion files appropriate for the ShakeMap tool (Wald et al. 2006) and event files. All files (which include information about the SN's performance during the event and the associated waveform data) are then stored on a portion of the ring buffer for future retrieval and analysis (see below).

Since the role of the LN is to "manage" a cluster of SNs, we must first emphasize that an $L N$ is still an $S N$, and, therefore,
(A)

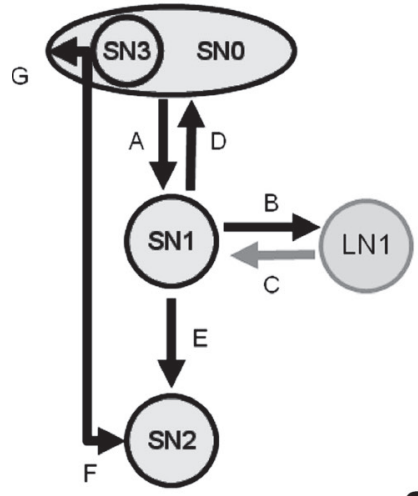

SN3 Disaster

Management Status

\section{SNO Monitoring Status}

SN1 Event Detection Status
(B)

SN2 Seismic Event Characterization Status

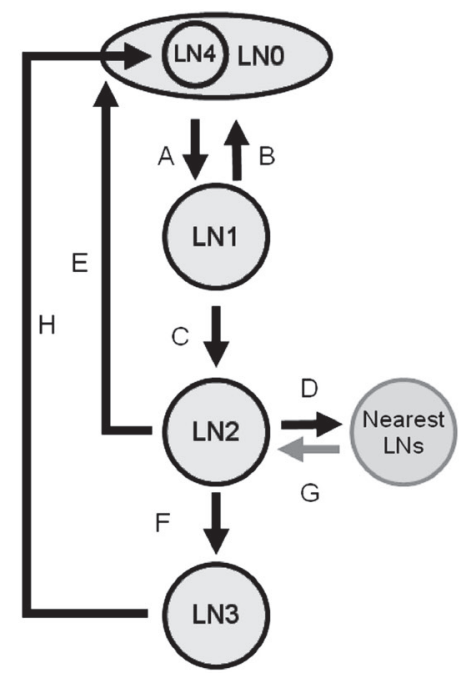

LNO Monitoring Status

LN1 pre-Alert Status LN2 Group-Alarm
Status

LN3 System Alarm

LN4 Disaster Management Status

- Figure 5. The state machines of (A) the sensing nodes and (B) leading nodes (see the text for a more detailed explanation of the various states). 
includes itself in all seismological activities. Its state machine (Figure 5B) therefore consists of the following:

- LN0, like SN0 for the SN, is the default or monitoring state, where the LN monitors the health of the SNs within its cluster. The only communications undertaken are with its SNs and the neighboring LNs. The network can be reorganized while all $\mathrm{LNs}$ are in this state.

- State LN1 (arrow A) is the pre-alert state or sensor alarm state, when an SN communicates to the LN that it (or the LN itself) has been triggered. From this point on, the cluster cannot be reorganized. If, however, after the expiring alert time fewer than $50 \%$ of the SNs within the cluster are triggered, the procedure mentioned above is carried out and the LN itself returns to LN0 (arrow B), with the previous trigger noted as a false alarm.

- State LN2 (arrow C) is the group alarm state (i.e. $\geq 50 \%$ of the SNs within the cluster have been triggered within the expiring alert time). The LN then informs its neighboring LNs (arrow D) that an event has possibly been detected. These LN are then in the state LN1 and inform their SNs (arrow F, Figure 5A). Again, if after a certain time (dependent upon the spacing between LNs) no other LNs indicate that an event has occurred, all LNs will return to LN0 (arrow E) and the event is noted as a false alarm.

- State LN3 (arrow F) is the alert state or system alarm state (i.e., two or more of the nearest LNs have indicated that an event has been detected, that is, they have also reached state LN2 (arrow G). At this point, an alert is issued throughout the network.

- State LN4 (arrow H) is the disaster management state, and like its equivalent in the $\mathrm{SN}$ state machine (SN3, Figure $5 \mathrm{~A})$, it is when the event is considered to have finished and the "all clear" has been given. The state of the network is checked, and, if necessary, some reorganization is undertaken.

\section{ROUTING PROTOCOLS}

The term "routing" refers to the selection of paths within a network along which data is sent from a source (the sensing units) to a sink (another sensor or an EN), although in a wireless environment, all nodes act concurrently as sources, sinks, and routers of data. Therefore, several possible paths usually exist that allow the transmission of data from one source to the same destination, and it is this that provides some protection against the failure of nodes. The independence of infrastructure, which makes WMNs easy to deploy, also leads to certain constraints and difficulties. For example, communication link quality within a WMN suffers from comparatively low bandwidth, which is further reduced by obstacles in and interference arising from the surroundings. Therefore, scalability is a major issue when developing routing protocols for WMNs.

SOSEWIN must be able to support the following network communication activities:

- Near-neighborhood communication over one or a few hops for the earthquake alarming protocol, where a mini- mum number of nodes must agree about the occurrence of an earthquake before an alarm is issued.

- The alarming itself would usually be done in two ways. The first is to route the alarm to the nearest GN and to the end users of the system. From this entity, the request for time-critical actions is then issued. The second is to communicate the alarm to every node (or at least LN) in the network. Usually both actions are taken simultaneously.

- In addition, during idle times or after an earthquake has destroyed a city's standard communications infrastructure, the network should be able to serve as a general purpose communications network.

SOSEWIN employs a proactive routing protocol, where every node (or in our case, every LN) has a map of the complete network topology, allowing data to be immediately sent along the optimal path. While keeping such information up-to-date is quite costly in terms of data transmission requirements, proactive protocols are better suited to early warning as delays in information transmission must be minimized, hence justifying the effort (i.e., heavier communications traffic arising from the continuous interrogation of the network and the associated increased power consumption) required to maintain up-todate knowledge of the optimal routes. As mentioned earlier, the protocol used is the OLSR protocol. This leads to each node having a routing table that describes the most efficient way to reach every other node. It makes use of advanced metrics, i.e., measurement methods, for the evaluation of a multi-hop path within the network.

However, message transmission must be as efficient as possible, particularly in limiting duplicate transmissions. This will be done by specifying certain sensing units within a cluster as multipoint relays (MPR). Each unit periodically broadcasts "Hello" messages to its direct neighborhood. These messages include the list of known neighbors, combined with the status of the quality of the connection to them. By knowing its two K-hop neighborhood, every node independently chooses a subset of the one K-hop neighborhood by which the complete two $\mathrm{K}$-hop neighborhood is reachable. This results in certain nodes being designated as MPRs, which, as seen in Figure 6, allows a reduction in transmissions when flooding the network, as only the MPRs need to rebroadcast a message to reach the complete two K-hop neighborhood. Every node also announces its chosen MPR, so that each node knows if it is an MPR or not. Nodes selected as MPR regularly flood the network with topology control (TC) messages at a defined interval (less frequently than "Hello" messages). These messages contain the link states of the nodes that selected this node as an MPR (the MPR selectors). By receiving these messages, a node therefore has enough information to locally reproduce the complete topology of the network. This enables a node to compute optimal paths to all known destinations, which in OLSR is done using Dijkstra's shortest path algorithm (Dijkstra 1959).

Other than the simple hop-count metric, which in no way reflects the link qualities within a path, the expected transmission count (ETX) metric takes the number of hops and the quality of transmission into account and in doing so improves 

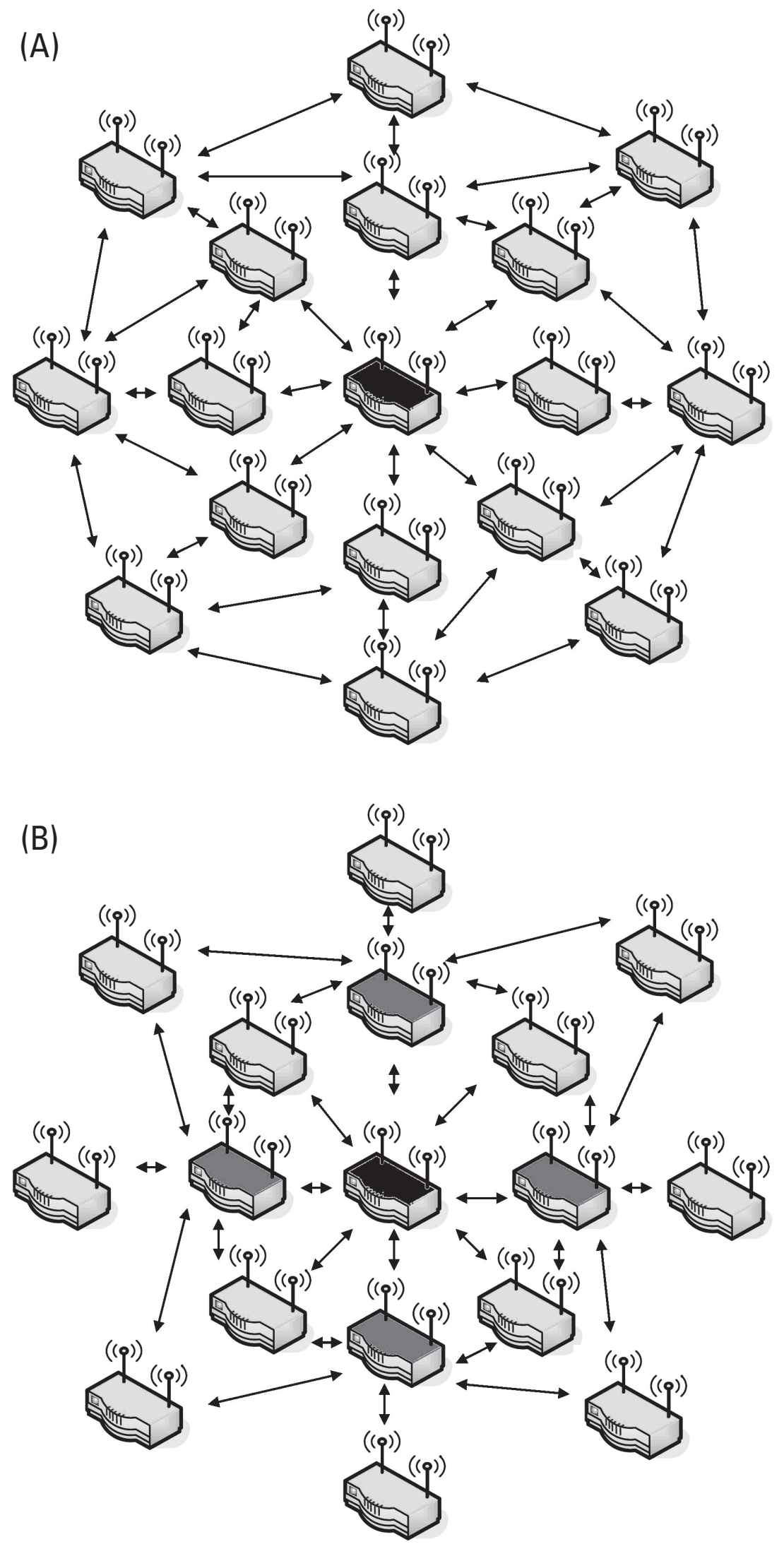

A Figure 6. How messages from a central node are distributed throughout a cluster. (A) Use of usual flooding, where all neighbors (light gray) of the central node (dark gray) broadcast its message. (B) Use of multipoint relays (medium gray), which are only a subset of the central node's neighbors responsible for rebroadcasting the message. 
the throughput and reliability of the network (De Couto et al. 2003; Nachtigall 2008).

\section{TEST DEPLOYMENT}

The first test-bed deployment of SOSEWIN was carried out in June 2008 with a network of 20 stations installed in the Ataköy district of Istanbul (Figure 7). Istanbul is a mega city (population 14 million) that is under significant risk from earthquakes. At its nearest point it is only tens of kilometers from the north Anatolian fault, along which there have been a number of large earthquakes over the past century, the most recent being the 1999 Izmit (17 August, $\mathbf{M}=7.4$ ) and 1999 Düzce (12 November, $\mathbf{M}=7.2$ ) earthquakes. In response to this seismic threat, and as discussed in the Introduction, there is currently in operation the IERREWS (Erdik et al. 2003). IERREWS consists of 100 strong-motion recorders in the densely populated areas of metropolitan Istanbul in dial-up mode and 10 online sensors located as close as possible to the Great Marmara fault for earthquake early warning. SOSEWIN would therefore complement the existing network, especially in its ability to "fill in gaps" (see Figure 1).

The choice of the Ataköy district was based on it being very well characterized from geotechnical and geophysical points of view. This is the result of a number of other facilities in the vicinity:

- The IERREWS strong-motion station AKUKO;

- The Ataköy vertical array site (administered by GFZ and KOERI; Parolai et al. 2009), consisting of accelerometric stations deployed at different depths in four bore-holes (25 $\mathrm{m}, 50 \mathrm{~m}, 75 \mathrm{~m}$, and $150 \mathrm{~m}$ deep) and at the surface;

- Single station and 2D-array measurements of microtremors administrated by GFZ (Picozzi et al. 2008).

The availability of so such information therefore makes Ataköy a very attractive candidate for our experimental SOSEWIN deployment (e.g., the $S$-wave velocity profile is estimated down to a depth greater than $200 \mathrm{~m}$, making it possible in the future for individual nodes to consider site response effects). In addition, proximity to one of the 10 IERREWS online sensors will allow us to evaluate the efficiency of SOSEWIN in undertaking EEW activities (i.e., event detection, issuing alarming messages).

The issues dealt with in this deployment include:

- Gaining experience in establishing such a network in an urban environment;

- Reliability of the communications between nodes;

- Reliability of the detection algorithms (i.e., setting different thresholds for different sites);

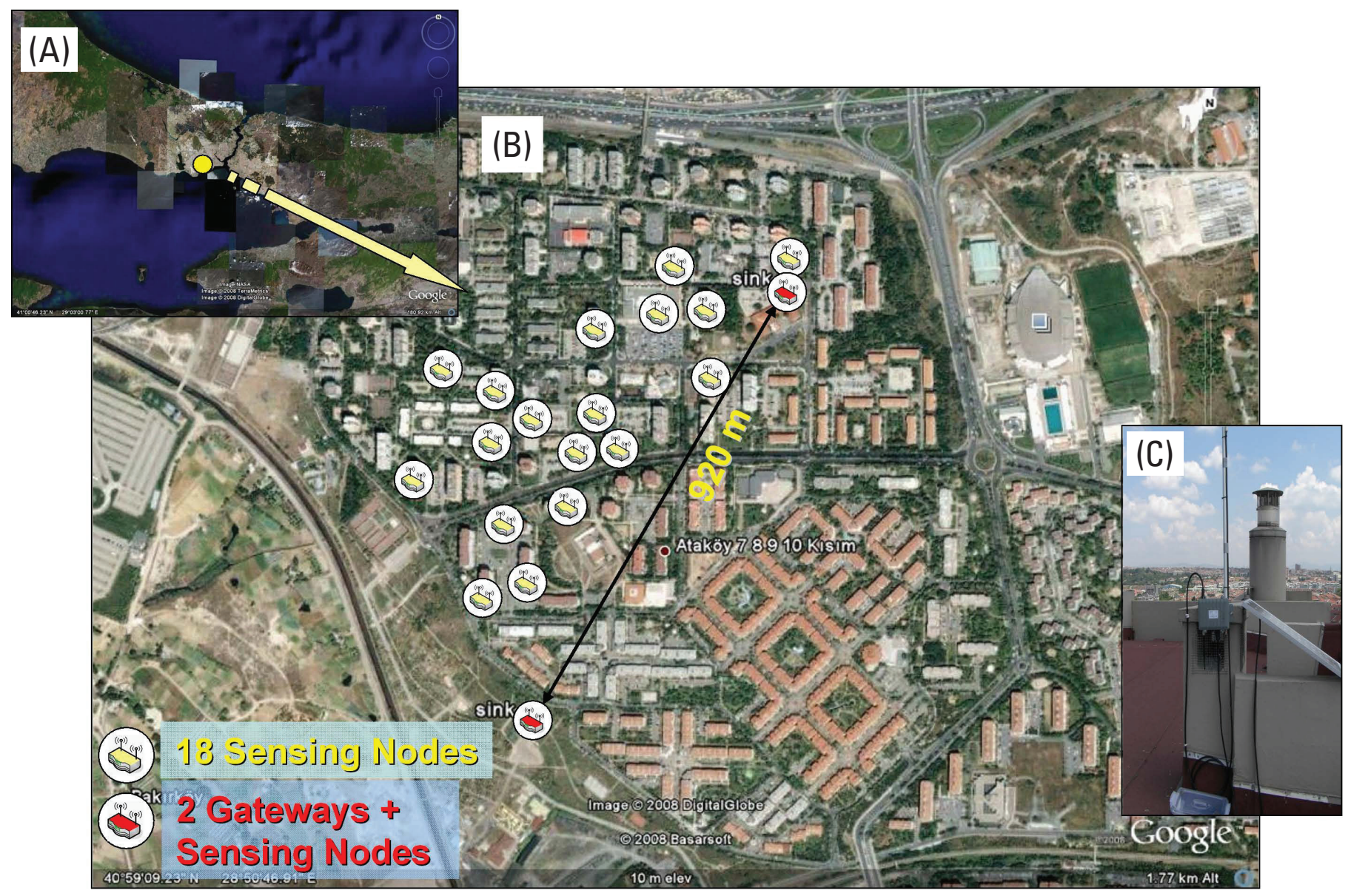

A Figure 7. (A) Location map of Istanbul showing the Ataköy district, where the test-bed SOSEWIN is situated. (B) The location of the sensing nodes and gateways for the test SOSEWIN in the Ataköy district, Istanbul. (C) A typical SOSEWIN sensing node installation. 
- Distinguishing between "local" events (traffic, thunderstorms, construction, etc.) and earthquakes of possible concern;

- Survivability of the system in the event of a sufficiently severe earthquake.

Figure 7 shows the location of the SNs, including the two GNs, which are $\mathrm{SN}$ s connected to the Internet via DSL. As shown in Figure $7(\mathrm{C})$, in order to better study the reliability of the communication between nodes, the SNs are at present all installed on the roofs of apartment buildings (on average 10 stories high) with clear line-of-sight views of other SNs. The nodes are powered sometimes by mains power with battery backup, or in some cases by solar panels, again with battery backup.

\section{DISCUSSION}

Since July 2008, a SeisComP Server at GFZ has collected SOSEWIN data in real time and distributed them to third parties (e.g., KOERI, Humboldt-Universität zu Berlin). In the present configuration, accelerometric data are transferred among SNs toward the two gateways following the SeedLink protocol (i.e., 512-byte Mini-SEED packets) and using a rate of $1 \mathrm{Mbps}$ in both the 2.4-GHz and (added later) the $5-\mathrm{GHz}$ unlicensed bands. As we have said before, the SeisComP performs automatic (near) real-time data processing (quality control, event detection). Thus, centralized early warning activities related to SOSEWIN are being carried out at GFZ. However, during this time, no significant seismicity has been observed close to Istanbul. For this reason, the preliminary tests of the test-bed network performance have focused on the various issues surrounding communications.

A first approach for the monitoring of the network performance quality is the aforementioned ETX metric (De Couto et al. 2003). This parameter describes the loss rate of a link between SNs, or in other words, the predicted number of data transmissions required to successfully transmit a packet from one $\mathrm{SN}$ to another. When the ETX equals 1, this represents a perfect connection, while increasing ETX values indicate less reliable links. Advantages of the ETX metric over other, more basic parameters (e.g., hop count metric) is that when nodes are not in direct contact and communications occur over a few hops, the ETX metric allows the selection of a route that is optimal with respect to the number of transmissions needed, while also excluding bad quality links from the path. An extensive description of the ETX and other metric parameters can be found in De Couto et al. (2003), Sombrutzki et al. (2006), and Nachtigall (2008).

In practice, the performance of the mesh network is evaluated by creating a graph of the network topology in terms of the ETX metric. Figure 8(A) shows an example of such a topology graph for the test-bed SOSEWIN. The quality of the $\mathrm{SN}$ connections is represented using three ranges of values for the ETX metric: from one to three is considered a good link, meaning that on average one to three transmissions are necessary to ensure successful transmittal of a 38-byte packet; the other ranges are three to six and greater than six. It is worth noting that SOSEWIN test-bed is effectively a high-quality mesh network, with all nodes able to communicate with some of the others with a low ETX value.

As a second test of the network performance, we verified which multi-hop path the network uses to connect the two gateways, which are separated by about $900 \mathrm{~m}$, and assessed the quality of the connection. Figure 8(B) shows the routes and the travel times for each hop followed by three 38-byte packets in the forward and backward directions between the gateways. In both cases, the communications between the two gateways need only four hops. Therefore, since the previously mentioned two K-hop and distance criteria are fulfilled, the two gateways could in principle be considered as two LNs. The delay in the transmission of packets varies at each link, dependent upon the dynamic nature of the WMN, but is generally below $50 \mathrm{~ms}$, indicating good-quality communication within SOSEWIN.

The final test we performed was to verify multi-hop packet transmission within SOSEWIN in the case where one LN must transmit information about a trigger to another LN (i.e.. the LNs correspond to the gateways). For this purpose, we selected the size of the warning packet as 520 bytes, which is realistic for a multi-parameter message. Moreover, the multihop transmission of the warning packet was repeated 200 times in each direction (i.e., forward, that is from gateway node 133.225 toward the other gateway node 133.222, Figures 7 and 8; and backward, from 133.222 to 133.225 ) between the LNs. Figure 9 shows a histogram that summarizes the results of this test. The distributions of travel times for the two directions look very similar, indicating the good quality in both directions of communication, hence the independence of the network-alarming performance with respect to the direction of the incoming threat. In total, $95 \%$ of the packets reached the target, and the average delay value of the classes representing the mode of the distributions is $80 \mathrm{~ms}$ and $74 \mathrm{~ms}$ for the forward and backward directions, respectively. The few outliers associated with larger delays (Figure 9) and the loss of some packets during the test is a clear example of the variability in communication link quality, due to unforeseeable interference arising within the WMN. Considering that the distance between the GNs is about $900 \mathrm{~m}$, the observed delays indicate that the warning packets traveled with an effective velocity of about $11.5 \mathrm{~km} / \mathrm{s}$ and $12.5 \mathrm{~km} / \mathrm{s}$ forward and backward, respectively, which is much faster than typical $P$-wave velocities (5-7 $\mathrm{km} / \mathrm{s}$ ).

\section{CONCLUSIONS}

In this article we have presented the development of a new approach for EEW. SOSEWIN represents innovative aspects with regard to existing EEWS. First, it is based on the use of new, low-cost wireless sensing units, specifically designed to form a dense WMN. These new sensors allow the performance of onsite, independent analysis of the ground motion and the real-time communication of estimated parameters. Second, following the philosophy of a decentralized network, SOSEWIN will perform early warning activities in a fully automatic way. 
(A)

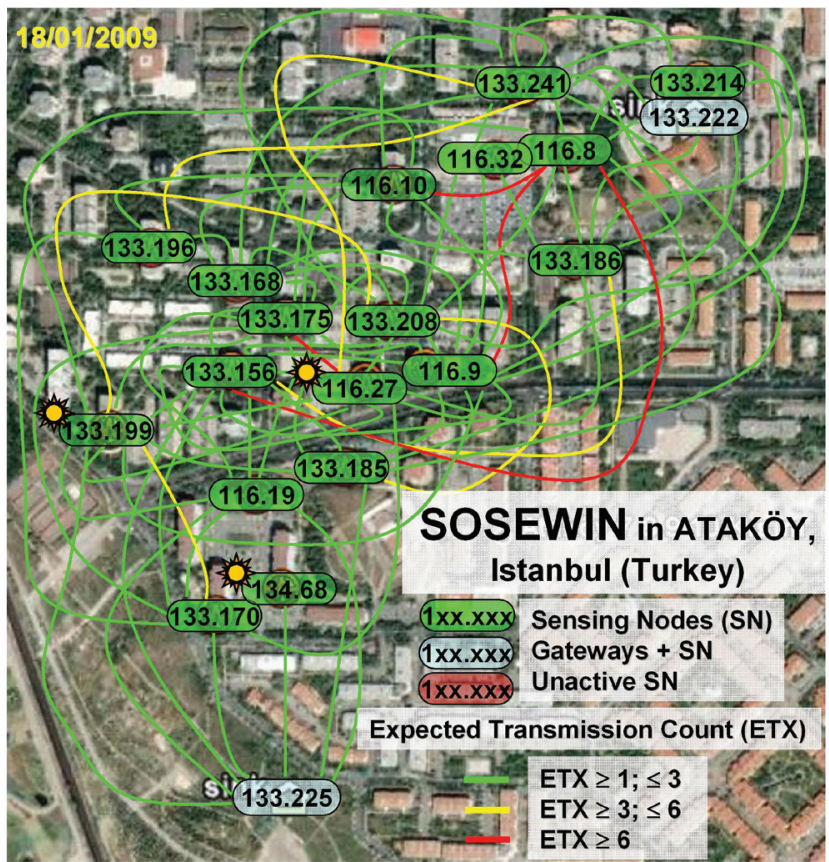

(B)

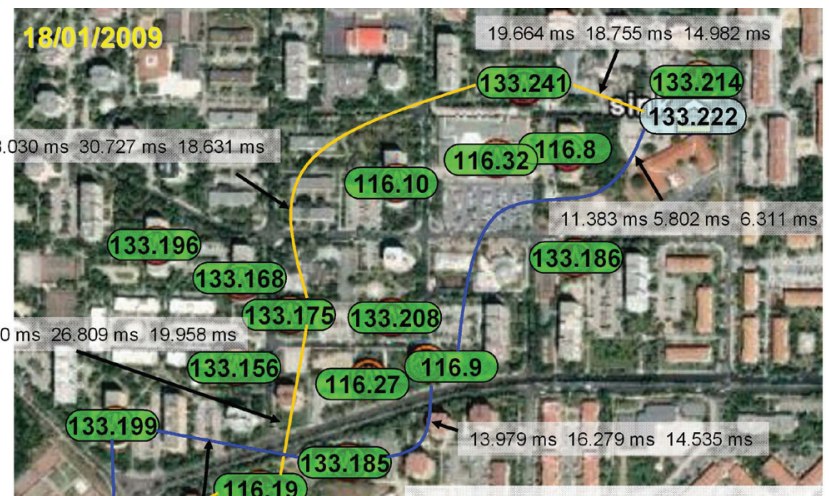

SOSEWIN in ATAKÖY, Istanbul (Turkey)

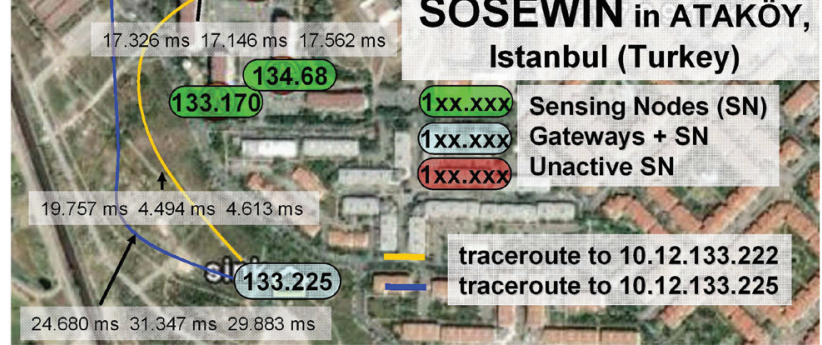

A Figure 8. Monitoring of SOSEWIN communication performance. (A) Link qualities. (B) Routing paths. The nodes 133.225 and 133.222 are the gateway nodes (see Figure 7). Note in the text that "forward" is from 133.225 to 133.222 and "backward"is from 133.222 to 133.225 .

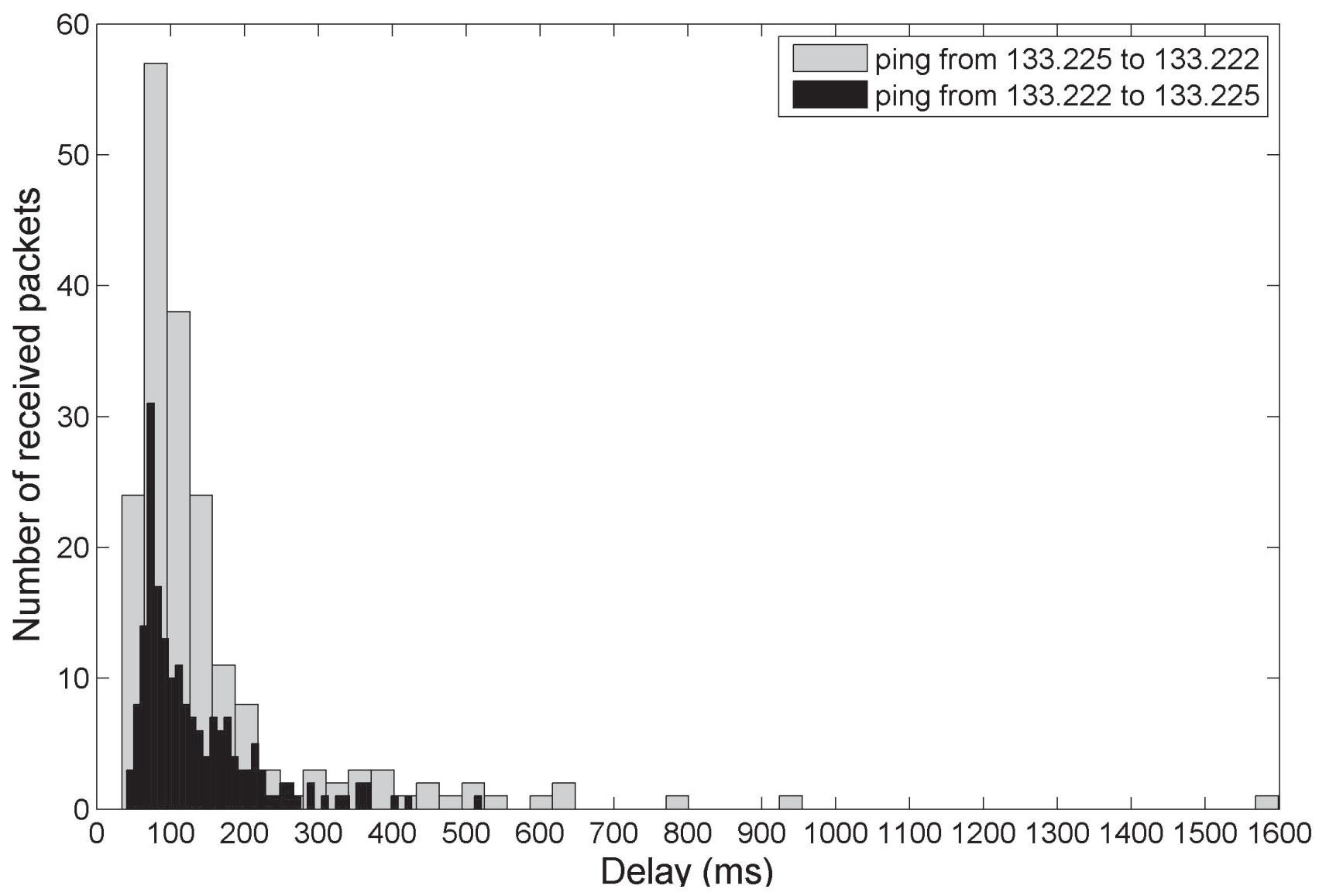

$\Delta$ Figure 9. Results of the test where 520-byte packages were transmitted 200 times between the two gateway nodes (in both directions, see Figure 8). As one can see, for the majority of time the packages arrived in less than $100 \mathrm{~ms}$. Note in the text that "forward" is from 133.225 to 133.222 and "backward" is from 133.222 to 133.225 . 
In practice, by means of dedicated algorithms, the decision to issue warnings will be made within the WMN itself, reducing the lead-time for early warning activities.

SOSEWIN represents the vision of what may be termed a people-centered EEWS. A long-term aim of SOSEWIN's development is that the $\mathrm{SNs}$ will evolve to a product that can be purchased by a range of end users, including the general public. This could potentially lead to networks made up of the order of thousands of nodes. The organization and routing protocols for such a network involve significant technical challenges. The planned employment of the OLSR protocol is based on it having been proved to be capable of operating with hundreds of nodes, while further optimizations like message grouping or the FishEye approach (which can be included in the current version used) potentially allow OLSR to be scaled up to thousands of nodes (Nguyen and Minet 2007). Such scalability is strongly dependent upon the real measured demands on the network traffic; hence, a great deal of effort will be expended in the appropriate simulations (e.g., Fischer et al. 2008).

Regarding the additional environmental sensor, any number of parameters for a variety of applications may be considered, including those not directly related to EEW (e.g., a noise sensor may be useful for monitoring traffic, thereby assisting with urban planning). Nonetheless, certain sensors may play a critical role in the post-earthquake response, e.g., temperature sensors may indicate fire, gas sensors could detect ruptured piping, and so forth. In addition, other sensors, such as threecomponent geophones, can be easily used in place of the accelerometers.

As mentioned earlier, providing information appropriate for ShakeMap-type output is an important product. This will potentially lead to higher resolution maps, allowing us to make neighborhood-scale loss assessments. While SOSEWIN is by no means intended to serve as a replacement for existing EEW networks made up of higher quality seismological units, it can nonetheless complement such systems while also allowing some degree of early warning to be established in areas where more traditional networks are economically prohibitive.

Since June 2008, a test-bed of 20 SOSEWIN sensors has been installed in Istanbul. Network activities are monitored, and data collected in real time, by a SeisComP server at GFZ. Until now, no significant seismic activity has been verified in the area. Preliminary tests of the network communication performance, however, give us confidence in the ability of this new kind of system in earthquake early warning.

\section{ACKNOWLEDGMENTS}

This work was supported by the SAFER (Seismic Early Warning for Europe, European Commission proposal no. 036935) and EDIM (Earthquake Data Information system for the Marmara Sea, Turkey, German Federal Ministry of Education and Research) projects. The establishment of the Istanbul network was carried out in partnership with Kandilli Observatory and Earthquake Research Institute. We thank the Bakirköy AKOM staff for their assistance in arrang- ing for and installing SOSEWIN in Istanbul. K.-H. Jäckel and Mike Hönig (Helmholtz-Zentrum Potsdam Deutsches GeoForschungsZentrum, Germany) developed the analog-digital converters (ADC) board.

\section{REFERENCES}

Arias, A. (1970). A measure of earthquake intensity. In Seismic Design for Nuclear Power Plants, ed. R. J. Hansen, 438-483. Cambridge, MA: MIT Press.

Böse, M. (2006). Earthquake early warning for Istanbul using artificial neural networks. PhD Thesis, Faculty of Physics, University of Karlsruhe, Germany.

De Couto, D. S. J., D. Aguayo, J. Bicket, and R. Morris (2003). A high-throughput path metric for multi-hop wireless routing. In Proceedings of the 9th Annual International Conference on Mobile Computing and Networking, September 14-19, 2003, San Diego, $C A$. New York: Association of Computing Machinery, 134-146.

Dijkstra, E. W. (1959). A note on two problems in connexion with graphs. Numerische Mathematik 1, 269-271.

Erdik, M., Y. Fahjan, O. Ozel, O. Alcik, A. Mert, and M. Gul (2003). Istanbul earthquake rapid response and early warning system. Bulletin of Earthquake Engineering 1, 157-163.

Espinosa Aranda, J. M., A. Jimenez, G. Ibarrola, F. Alcantar, A. Aguilar, M. Inostroza, and S. Maldonado (1995). Mexico City seismic alert system. Seismological Research Letters 66 (6), 42-53.

Evans, J., R. Hamstra, C. Kündig, P. Camina, and J. Rogers (2005). TREMOR: A wireless MEMS accelerograph for dense arrays. Earthquake Spectra 21 (1), 91-124.

Fischer, J., F. Kühnlenz, and K. Ahrens (2008). Model-based development of self-organizing earthquake early warning system. In Joint ITU-T and SDL Forum Society Workshop on "ITU System Design Languages," Geneva, Switzerland, http://www.itu.int/dms_pub/ itu-t/oth/06/18/T06180000010022PDFE.pdf.

Heinloo, A. (2000). SeedLink design notes and configuration tips; http://geofon.gfz-potsdam.de/geofon/seiscomp/seedlink.html.

Holland, A. (2003). Earthquake data recorded by the MEMS accelerometer. Seismological Research Letters 74 (1), 20-26.

Hons, M., R. Stewart, D. Lawton, and M. Bertram (2008). Field data comparisons of MEMS accelerometers and analog geophones. The Leading Edge (July 2008), 896-902.

Horiuchi, S., Y. Horiuchi, S. Yamamoto, H. Nakamura, C. Wu, P. A. Rydelek, and M. Kachi (2009). Home seismometer for earthquake early warning. Geophysical Research Letters 36; doi: 10.1029/2008GL036572.

Horiuchi, S., H. Negishi, K. Abe, A. Kamimura, and Y. Fujinawa (2005). An automatic processing system for broadcasting earthquake alarms. Bulletin of the Seismological Society of America 95 (2), $708-718$.

Husker, A., I. Stubailo, M. Lukac, V. Naik, R. Guy, P. Davis, and D. Estrin (2008). WiLSoN: The wirelessly linked seismological network and its application in the middle American subduction experiment. Seismological Research Letters 78 (3), 438-443.

Iglesias, A., S. Singh, M. Ordaz, M. Santoyo, and J. Pacheco (2007). The seismic alert system for Mexico City: An evaluation of its performance and strategy for its improvement. Bulletin of the Seismological Society of America 97 (5), 1,718-1,729.

International Strategy for Disaster Reduction (2005). Hyogo Framework for Action 2005-2015: Building the Resilience of Nations and Communities to Disasters. World Conference on Disaster Reduction, 18-22 January 2005, Kobe, Hyogo, Japan.

Ionescu, C., M. Böse, and F. Wenzel (2007). An early warning system for deep Vrancea (Romania) earthquakes. In Earthquake Early Warning Systems, ed. P. Gasparini, G. Manfredi, and J. Zschau, 343-349. Berlin and Heidelberg: Springer. 
Kanamori, H., P. Maechling, and E. Hauksson (1999). Continuous monitoring of ground-motion parameters. Bulletin of the Seismological Society of America 89 (1), 331-316.

Nguyen, D., and P. Minet (2007). Scalability of the OLSR protocol with the FishEye extension. In Proceedings of the Sixth International Conference on Networking, April 22-28, 2007, Sainte-Luce, Martinique, IEEE, 88, doi:10.1109/ICN.2007.80.

Nachtigall, J. (2008). Earthquake information system using wireless mesh networks. Master's thesis, Humboldt-Universität zu Berlin, Geographisches Institut, Berlin, Germany, 138 pps.

Olson, E. L., and R. M. Allen (2005). The deterministic nature of earthquake rupture. Nature 438, 212-215.

Parolai, S., A. Ansal, A. Kurtulus, A. Strollo, R. Wang, and J. Zschau (2009). The Ataköy vertical array (Turkey): Insights into seismic wave propagation in the shallow-most crustal layers by waveform deconvolution. Geophysical Journal International. doi: 10.1111/j.1365-246X.2009.04257.x

Picozzi, M., A. Strollo, S. Parolai, E. Durukal, O. Özel, S. Karabulut, J. Zschau, and M. Erdik (2008). Site characterization by seismic noise in Istanbul, Turkey. Soil Dynamics and Earthquake Engineering 29(3), 469-482. doi:10.1016/j.soildyn.2008.05.007.

Savazzi, S., and U. Spagnolini (2008). Wireless geophone networks for high-density land acquisition: Technologies and future potential. The Leading Edge (July 2008), 882-886.

Schweitzer, J., J. Fyen, and S. Mykkeltveit (2002). Seismic arrays. In IASPEI New Manual of Seismological Observatory Practice, ed. P. Bormann, chapter 9. Potsdam, Germany: GeoForschungsZentrum Potsdam.

Sleeman, R., and T. van Eck (1999). Robust automatic P-phase picking: An on-line implementation in the analysis of broadband seismogram recordings. Physics of the Earth and Planetary Interiors 113 $(1-4), 265-275$.

Sombrutzki, R., A. Zubow, M. Kurth, and J.-P. Redlich (2006). Selforganization in community mesh networks-The Berlin RoofNet. In First Workshop on Operator-Assisted (Wireless Mesh) Community Networks, 18-19 September 2006, Berlin. J.P. Redlich ed., 1-11.

Trnkoczy, A. (2002). Understanding and parameter setting of STL/ LTA trigger algorithm. In IASPEI New Manual of Seismological Observatory Practice, ed. P. Bormann, information sheet IS 8.1. Potsdam, Germany: GeoForschungsZentrum Potsdam.

Trnkoczy, A., J. Havskov, and L. Ottemöller (2002). Seismic networks. In IASPEI New Manual of Seismological Observatory Practice, ed. P.
Bormann, chapter 8. Potsdam, Germany: GeoForschungsZentrum Potsdam.

Torvalds, L. (2007). The Linux kernel; http://www.kernel.org.

United Nations (2006). Global Survey of Early Warning Systems. Gaps and OpportunitiestowardsBuildinga Comprehensive GlobalEarly Warning System for All Natural Hazards. Report prepared at the request of the Secretary-General of the United Nations. http://www.unisdr.org/ preventionweb/files/3612_GlobalSurveyofEarlyWarningSystems.pdf

Wald, D. J., B. C. Worden, V. Quitoriano, and K. L. Pankow (2006). ShakeMap Manual: Technical Manual, Users Guide, and Software Guide; http://pubs.usgs.gov/tm/2005/12A01/pdf/508TM12-A1.pdf.

Wu, Y.-M., and H. Kanamori (2005). Experiment on an onsite early warning method for the Taiwan early warning system. Bulletin of the Seismological Society of America 95 (1), 347-353.

Wu, Y.-M., and H. Kanamori (2008). Development of an earthquake early warning system using real-time strong motion signals. Sensors $8,1-9$.

Wu, Y.-M., and T.-L. Teng (2002). A virtual subnetwork approach to earthquake early warning. Bulletin of the Seismological Society of America 92 (5), 2,008-2,018.

Wurman, G., R. M. Allen, and P. Lombard (2007). Toward earthquake early warning in northern California. Journal of Geophysical Research 112; doi: 10.1029/2006JB004830.

Zhang, H. J., C. Thurber, and C. Rowe (2003). Automatic P-wave arrival detection and picking with multiscale wavelet analysis for single component recordings. Bulletin of the Seismological Society of America 93 (5), 1,904-1,912.

\section{Department of Spatial Sciences Curtin University of Technology Perth, Western Australia, Australia k.fleming@curtin.edu.au}

(K. F.)

\section{Helmboltz-Zentrum Potsdam Deutsches GeoForschungsZentrum Telegrafenberg 14473, Potsdam, Germany picoz@gfz-potsdam.de}

(M. P.) 